
45

FINAL REPORT PROJECT NO. IS-1275-87

\title{
Characterization of Citrus Viroids as Potential Dwarfing Agents of Citrus
}

M. Bar-Joseph, J. Semancik

(1) A)

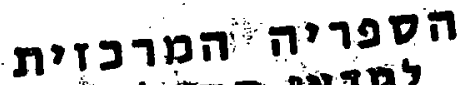

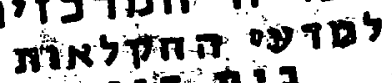

$\frac{630.72}{B A R / B A R}$

BAR/BAR

2nd copy 


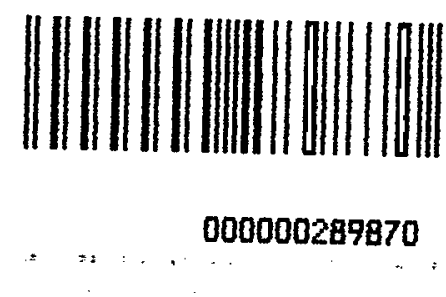


BARD

P.O. Box 6

Bet Dagan, ISRAEL

BARD Project No. IS-1275-87

Title of Research Project:

Characterization of citrus viroids as potential dwarfing agents of citrus

Names of Investigators:

Principal Investigator: M. Bar-Joseph

Cooperating Investigator(s): J.S. Semancik

Name and Address of Affiliated Institutions:

Principal Institution: The Volcani Center, Agricultural Research Organization, Bet Dagan Israel

Cooperating Institution: University of California, Riverside CA 92521, U.S.A.

Project's starting date: November, 1988

Type of Report: Final

\section{Signatures:}

Principal Investigator

Moshe Bar-Joseph

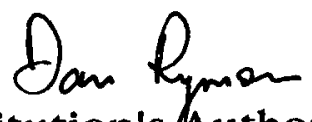

Institution's Authorizing Official

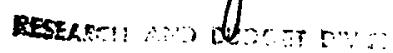




$$
\begin{aligned}
& \frac{630.72}{\text { BAR/BAR }}: 576.64: 634.3: 574.973 .2: 581.1: 632: \\
& 2^{\text {nd } C}
\end{aligned}
$$




\section{Table of contents}

Cover Page-1.-1 1

Table of contents- 2

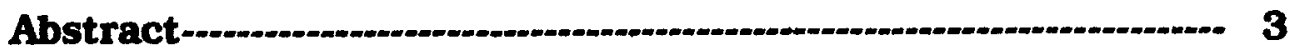

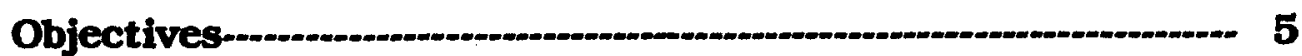

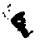

Description of research results-_- 6

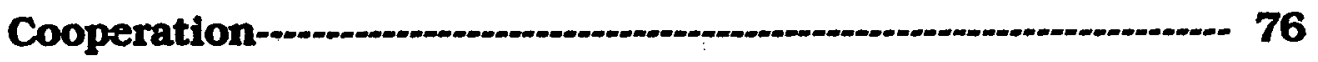

Evaluation of research achievements-_._.-. 76 


\section{Abstract}

Characterization of citrus viroids as potential dwarfing agents of citrus

This project has addressed two main questions:

(1) What is the nature of the most important citrus viroids used for dwarfing citrus trees in Israel, and

(2) What is the relationship between these viroids and the blological response they elicit .

The citrus viroids which compose the graft transmissible dwarfing (GTD) agent \# $225 \mathrm{~T}$, the main source of inoculum for dwarfing grapefruit trees in Israel were characterized. GTD \#225 was found to contain at least five CVds with estimated sizes of 284, 295, 299, 318 and 371 bases. Two mildly dwarfed subclones (225-M and 225-VM) lacked the CEVd band, but each contained three viroids of about 284, 295, 318 and 284, 295 and 299 nucleotides respectively. Segregation and exclusion of some CVds from the GTD inoculum were found to cause the inconsistent dwarfing effects. The CVd complexes rarely segregated when Ethrog citron (Citrus medica) was used as the inoculum source and recommendations were made to use it for more consistent dwarfing Previous host range studies have shown that the CEVd present in CVd complexes could be separated by inoculating Gynura aurantiaca plants. The 284 and 299 base CVds could be transmitted and separated by serlal transfer to cucumber and Benincasa hisplda. The host range of $318 \mathrm{~b} C V d$, classified as CV-Ib was considered to be restricted to Citrus spp. In this work we have separated the CVd of 318 bases by graft transmission on avocado plants. A method was developed for the use of the polymerase chain reaction for the amplification of cDNA from newly found RNA species. Using this method we have amplifled and cloned the $318 \mathrm{~b}$ CVd which we named citrus bent leaf viroid (CBLVd). Sequence analysis of CBLVd shows that it is a novel chimeric viroid species constituted from a part of the central conserved region (CCR) of the apple scar skin viroid (ASSVd), and part of the pathogenic (P) region and the left terminal region of CEVd. Sequence analysis of the $371 \mathrm{CVd}$ from GTD\#225 has revealed deference's in 25 positions compared to the 
type strain (CEVd-A), most of these sequence changes were located in two CEVd domains, the pathogenic $(P)$ and variable $(V)$ domain. Sequence analysis of the $299 \mathrm{CVd}$ revealed that this CVd is a sequence variant of hop stunt viroid (HSVd), differing from the Japanese HSVd type strain (297 nts) in seven positions. Sequence analysis of CVd-IV ( $284 \mathrm{nts}$ ) revealed that it is a novel chimeric viroid, with most of its right hand part and it's CCR resembling CEVd, whereas the left terminal region was very similar to that of HSVd (the sequence analyses of the last two viroids were conducted in Prof. Saenger's Laboratory). Interactions among the group II CVds were investigated. Interference was demonstrated between CVd-IIa, a mild exocortis agent and CVd-IIb the severe cachexia agent was demonstrated. These results offer a new approach for the control of the cachexia disease by "viroid interference". For the stable insertion of the CEVd into plants the CDNA of CEVd was cloned between the 35S promoter and terminator and introduced to a mini Ti (pGA 482) of Agrobacterium tumefaciens, The bacteria were used for transformation of tobacco and tomato plants. The transgenic tomato plants were found to contain transcripts of CEVd. Experiments almed to obtain a stabile transformed inoculum source of citrus are in progress. 


\section{Original Objectives}

(a) to determine and compare the citrus viroid profiles present in Israel and the USA.

(b) to characterize the specific citrus viroid or viroid mixtures responsible for the dwarfing reaction.

(c) To establish glass house and field trials to evaluate the effect of some of the different citrus viroids on various scion/ rootstock combinations of commercial citrus

(d) to develop probes to the different citrus viroid groups to determine the molecular relationships between groups. 


\section{A general introduction on dwarfing by citrus viroids}

The following account, cited with major changes (Golomb ,1988) describes the history of citrus dwarfing in Israel: Viroids have been used for decades as beneficial dwarfing agents of citrus trees in high density plantings (HDP) of citrus in Israel. The traditional citrus industry in this country was based on the observation that the "Shamouti"(Jaffa) orange (C. sinensis) grafted on the Palestine (Indian) Sweet lime (PSL) (C. limettiodes) rootstock gave in sandy soils trees with excellent performance. Trees of this combination had a swift growth at their start and where early bearers, but gradually their growth tended to slow down and the tress became of compact size but kept a steady production for about 25-35 years. Until the mid fifties, for almost a century, such citrus groves that had been densely planted $(62-110$ trees $/ 1000 \mathrm{~m})$ and manually cultivated and irrigated, enjoyed the full benefits of what we consider now as HDP.

When citrus had to be planted in heavy solls, the Sour orange ( $C$. aurantium) rootstock was used to replace the PSL rootstock in order to prevent root-rot gummosis problems caused by Phytophtora $\mathrm{sp}$. The resulting "Shamouti" scions developed large, late and less productive trees with a high tendency towards late and alternate bearing.

These observations led Mendel (1968) to suggest the use of 'old clone' budwood infected with certain uncharacterized graft transmissible disease (GTD) agents as a mean to obtain early bearing HDP plantations on GTD sensitive rootstocks. Similar observations were made in Australia leading to several large scale fleld experiments (Bevington and Bacon, 1977) that have further substantiated this rather unorthodox concept.

Preliminary trials carried out during the years 1965-1980 in about 25 small HDP observation plots (0.3-1.0 ha each) with different citrus varieties carrying different GTD sources grafted on trifoliate orange ( Poncirus trifollata) and Troyer citrange ( $\underline{P}$. trifollata $\times \underline{C}$. sinensis) rootstocks gave inconsistent results. Some of these experiments were conducted at experimental farms but the majority, in commercial groves. 
Many of the latter were a clear fallure and eventually taught an invaluable lesson that trees of the above combinations when inoculated with GTD will only be productive at absolutely good farming conditions. Fortunately, with progress made in irrigation, fertilization and weedcontrol the currently used horticultural practices enable now the application of GTD dwarfing of HDP groves at the ordinary farming level.

Following these observations a large scale field testing plot aimed to analyze the effects of two GTD sources, two inoculation times on four citrus varietles grafted on two types of trifoliate oranges was established in 1981 at the Hulla Experimental farm Northern Galllee. Results gathered from these experiments over a period of ten years will be summarized in a separate paper (Amital, Ashkenazl, Oren, Zamir and Bar-Joseph, in preparation).

A brief illustration of the horticulture and economic advantages of the HDP practice resulting from the these experiments is given in Fig 1 which shows the cumulative yields obtained over a period of 9 years after planting of GTD \# $225 \mathrm{~T}$ inoculated Star Ruby grapefruit trees grafted on trifoliate rootstock at two HDP conditions of 100 and 166 trees $/ 1000 \mathrm{~m}$ compared to the conventionally grown trees with 40 tree/ $1000 \mathrm{~m}$. Similar results have been now obtained from a number HDP commercial groves (Amital, Ashkenazi, Oren, Zamir and Bar-Joseph, unpublished)

It is thus satisfying to mention that while pursuing this project most of the new plantings with about 400.000 trees in the northern Gallee have been densely planted and either GTD dwarfed or prepared for such dwarfing.

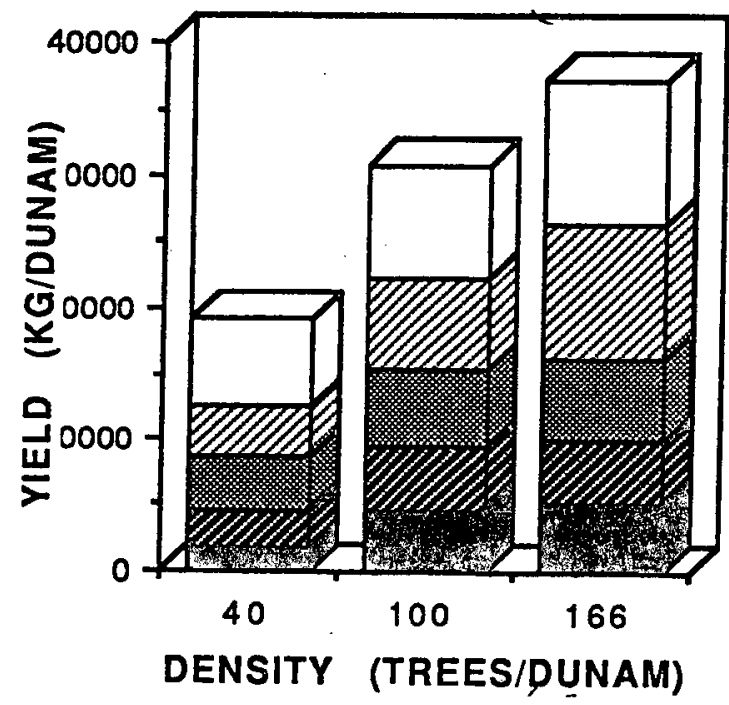

Fig 1 The cumulative yields of two viroid inoculated Star Ruby grapefruit HDP plantings with 100 and 166 trees /1000m , compared to a regularly spaced planting. Yield records starting after 3-years from planting, 
Characterization of viroids associated with GTD \#225 the main source for dwarfing grapefruit trees in Israel

During the last decade several hundred thousand grapefruit trees have been increased or graft inoculated with budwood collected from a grapefruit tree designated GTD \# $225 \mathrm{~T}$, located at the Acco Experiment Station or with budwood collected from some of the of-springs of this tree (Ashkenazi, personal communication). The selection of GTD \#225 as the main source for dwarfing was empirical and based on observations indicating the satisfactory performance and acceptable form of trees budded or inoculated with budwood from this tree.

Several obstacles associated with the use of GTD \#225 budwood are addressed in the following papers. The results provide evidence for the segregation and exclusion of some of the citrus viroids (CVds) which form the graft transmissible disease complex responsible for the dwarfing effects of sensitive stionic combination.

The inconsistent presence of one or more of the CVds in certain buds collected from GTD \# 225 provided a plausible explanation to one of the major drawbacks of the GTD inoculation, that's the considerable variation in tree size and performance often observed in HDP plantings treated with GTD \# 225 and other sources of inoculum.

The practical implications of these findings include the need to change the inoculum sources from the traditional grapefruit source to other plants which are more persistent in hosting and transmitting all the CVds included in this complex.

Experiments are currently underway in flve locations including the Hula Experiment station and four commercial groves to evaluate the use of GTD \# $225 \mathrm{~S}$ and other isolated CVd sources increased in three other inoculum source plants ( Ethrog, Rangpur lime and Volcameriana). The results of these experiments will only be available after additional 3-4 years. 
A comprehensive field testing of citrus virolds with a total of 713 trees has been undertaken since the fall of 1991 at the CRC Experiment station, Riverside. The outline of this project is presented bellow.

A) Citrus Viroid Field Test of Commercial Cultivars and Rootstocks.

Commercial cultivars of navel and valencia oranges, lemons, and grapefruit will be propagated on the seven rootstock selections listed Eelow. Citrus viroid inoculations and control treatments are as described. Evaluation of the 448 trees in this trial will be made by standard parameters of determining tree vigor and performance.

SCION VARIETIES

Atwood Navel

Lane Late Nave1

Campbell Valencia Cutter Valencia

Allen Eureka Lemon Limoneira $8 \mathrm{~A}$ Lisbon

Rio Red Grapefruit Melogold Grapefruit

\section{ROOTSTOCKS}

\section{Carrizo Cftrange}

C-35 Citrange

Rubidoux Trifoliate

African Shaddock $X$ Rubidoux Trifoliate

Schaub Rough Lemon

Yuma Ponderosa Lemon

Citrume 701452

The following treatments will be included in the trial and repeated on ail of the scion/rootstock combinations:

1) Inoculated with severe Citrus Exocortis Virotd (E811).

2) Inoculated with a mild Citrus Viroid (CVd-IIa) found in the Parent Washington Navel (EB18).

3) Inoculated with a viroid mixture of representatives of Citrus virotd Group I, -II, -111 , and -IV.

4) Heal thy control.

B) Cachexia "Interference" Trial.

We have observed in citron that the titer of the viroid inducing severe cachexia disease can be reduced by co-inoculation with a related viroid. This trial is designed to determine:

a) If a similar virotd "interference" can be observed in commercial citrus, and

b) if this procedure can be employed to moderate the expression of the cachexia disease in the field.

Two sources of tangelo and mandarin will be employed with two different rootstocks for each scion for a total of 128 trees in this trial.

Scion Varieties

Minneola Tange 10

Orlando Tangelo

\section{Rootstocks}

Carrizo Citrange

Trifolịate Orange

Satsuma Mandarin

Clementine Mandarin

The essence of this test will involve preinoculation with the mild ' viroid isolate and a secondary inoculation with the severe cachexia agent about one year later ("c" below). Treatments "a", "b", and "d" will aiso be included is controls:

1) Inoculated with mild CV-IIa. (EBIB).

2 Inoculated with severe cachexia CV-IIb (Ca902).

3 Inoculated with CV-Ila and one year later with $\mathrm{CV}$-IIb.

4) Healthy controls.

An evaluation of "viroid interference" will be' made by direct observations for any evidence of cachexta disease as well as an analysis of the tree performance. 


\section{Description of collaboration}

During the research period continuos cooperation was maintained between the two laboratories via regular and electronic mall.

A joint research paper describing the CVd segregation phenomena was published. In addition several visits between the two laboratories took place . Prof. Semancik visited twice the $S$. Tolkowsky laboratory and had a first hand opportunity to see the experimental and commercial viroid dwarfing plots in the Galilee. Prof. Bar-Joseph visited at the University of California Riverside. During these visits, results and experimental designs were discussed.

\section{Evaluation of the research achievements}

GTD \# 225 had been intensively used in certain parts of Israel for dwarfing citrus trees during the last decade. This project has enabled for the first time an SPAGE analyses of the citrus viroids which are present and GTD \#225 and several other dwarfing complexes and lead to the molecular characterization of four of these CVds.

Two viroids CVd IV and CBLVd were found to be new natural chimeric molecules between CEVd and HSVd ( CVd IV) and between CEVd and ASSVd ( CBLVd), Sequencing of two other CVds revealed that the $371 \mathrm{~b}$ CVd was different in 25 bases from the type strain (CEV-A) and probably belongs to CEV Class $B$ and the $299 \mathrm{~b}$ CVd is a sequence variant of HSVd differing from the Japanese type strain (297b) in seven positions.

Segregation and exclusion of some CVds from the GTD inoculum was found to cause the inconsistent dwarfing effects, often causing serious difficulties under practical conditions. Following our observation that the CVd complexes rarely segregated in Ethrog citron, recommendations were made to use Ethrog as inoculum source. Large scale field studies are now in progress in order to demonstrate the advantages of using Ethrog and some other host plants as inoculum sources. 
Separation of CBLVd (318b) from the other CVds was achieved by graft transmission to avocado. Host range studies were conducted under contained conditions in order to evaluate the pathogenicity of CBLVd to different avocado cultivars. None of the commercial avocado varieties was found to show adverse reactions following CBLVd inoculation and experiments are underway to locate a CBLVd sensitive avocado relative to be used eventually as rootstock for dwarfing avocado trees.

Interactions among the group II CVds were investigated. Interference between CVd-IIa, a mild exocortis agent and CVd-IIb the severe cachexia agent was demonstrated. These results offer a new approach for the control of the cachexia disease by "viroid interference".

A vector enabling the stable insertion of a monomeric CEVd CDNA flanked with transcription signals was used for stable transformation of tobacco and tomato plants. The transgenic tomato plants were found to contain infective transcripts of CEVd. Experiments aimed to obtain a stabile transformed inoculum source of citrus are in progress.

Finally it is satisfying to mention that while pursuing this project most of the new plantings with about 400.000 trees in the northern Galilee have been densely planted and either GTD dwarfed or prepared for such dwarfing. For many of these newly dwarfed trees inoculum sources developed from this project will be used. 